\title{
Pulsed helium ion beam induced deposition: A means to high growth rates
}

\author{
Paul F. A. Alkemade ${ }^{a}$ and Hozanna Miro \\ Kavli Institute of Nanoscience, Delft University of Technology, Lorentzweg 1, 2628 CJ Delft, The Netherlands \\ Emile van Veldhoven and Diederik J. Maas \\ TNO Van Leeuwenhoek Laboratory, 2628 CK Delft, The Netherlands
}

Daryl A. Smith and Philip D. Rack

Materials Science and Engineering Department, University of Tennessee, Knoxville Tennessee, 37996-2200

(Received 4 July 2011; accepted 5 October 2011; published 3 November 2011)

\begin{abstract}
The sub-nanometer beam of a helium ion microscope was used to study and optimize helium-ion beam induced deposition of $\mathrm{PtC}$ nanopillars with the $\left(\mathrm{CH}_{3}\right)_{3} \mathrm{Pt}\left(\mathrm{C}_{\mathrm{P}} \mathrm{CH}_{3}\right)$ precursor. The beam current, beam dwell time, precursor refresh time, and beam focus have been independently varied. Continuous beam exposure resulted in narrow but short pillars, while pulsed exposure resulted in thinner and higher ones. Furthermore, at short dwell times the deposition efficiency was very high, especially for a defocused beam. Efficiencies were measured up to 20 times the value for continuous exposure conditions. The interpretation of the experimental data was aided by a Monte Carlo simulation of the deposition. The results indicate that two regimes are operational in ion beam induced deposition (IBID). In the first one, the adsorbed precursor molecules originally present in the beam interaction region decompose. After the original precursor layer is consumed, further depletion is averted and growth continues by the supply of molecules via adsorption and surface diffusion. Depletion around the beam impact site can be distinguished from depletion on the flanges of the growing pillars. The Monte Carlo simulations for low precursor surface coverage reproduce measured growth rates, but predict considerably narrower pillars, especially at short dwell times. Both the experiments and the simulations show that the pillar width rapidly increases with increasing beam diameter. Optimal writing strategy, good beam focusing, and rapid beam positioning are needed for efficient and precise fabrication of extended and complex nanostructures by He-IBID. (C) 2011 American Vacuum Society. [DOI: 10.1116/1.3656347]
\end{abstract}

\section{INTRODUCTION}

Developments in nanotechnology depend on techniques for making structures on the nanometer scale with sufficient precision, purity, and speed. Ion beam induced deposition (IBID) is one method to directly write or grow nanostructures. The recent introduction of the helium ion microscope with a sub-nanometer probe beam ${ }^{1}$ provided a new means for helium ion beam induced deposition (He-IBID). Initial experiments showed that He-IBID is a good alternative for the relatively slow electron beam induced deposition (EBID) and the lowerresolution gallium ion beam induced deposition. ${ }^{2-4}$ The narrow probe size, the narrow interaction volume of helium ions in materials, and the relatively large number of secondary electrons especially allow for high spatial resolution nanofabrication at relatively moderate speeds. The main bottleneck in high-resolution He-IBID is the transport of precursor molecules to the sites of deposition. Because of the high intensity of the ion beam, precursor depletion quickly occurs, causing reduced growth efficiencies, especially at the beam impact site, as seen in Fig. 1. Pulsing ion beams that allow precursor refreshment to the depleted regions is one remedy against diminished efficiencies.

In this work, we grow arrays of nanopillars by He-IBID with a cycling, or pulsing, focused helium ion beam. Varia-

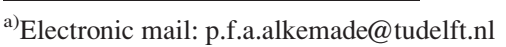

tions in beam current, beam dwell times, beam focus, and precursor refresh times allow optimization of the growth rate. This type of experiment provides quantitative data of the basic physical and chemical processes. ${ }^{5}$ Because most of these processes are very complex, we use the "EnvisION" Monte Carlo simulation model ${ }^{6}$ to aid in the interpretation of the experimental observations.

\section{EXPERIMENT}

The experiments were performed in a Carl Zeiss Orion ${ }^{\mathrm{TM}}$ Plus scanning helium ion microscope (HIM), equipped with an OmniGIS unit from Omniprobe. We have used the same beam and gas conditions as in Refs. 3 and 4. The angle between the nozzle and the surface normal was $65^{\circ}$ (in Ref. 3 , an angle between the nozzle and the surface normal of $25^{\circ}$ was mentioned; this is an error; actually it is $65^{\circ}$ ), the opening of the nozzle is $500 \pm 50 \mu \mathrm{m}$ wide, and the nozzle's center is $600 \pm 100 \mu \mathrm{m}$ above the substrate surface. The precursor gas was $\left(\mathrm{CH}_{3}\right)_{3} \mathrm{Pt}\left(\mathrm{C}_{\mathrm{P}} \mathrm{CH}_{3}\right)$ and the substrate material was $\mathrm{Si}$ with a native oxide. We estimate the pressure above the beam impact site to be $1.3 \times 10^{-2}$ mbar. $^{3}$ Arrays of pillars at $1 \mu \mathrm{m}$ separations were grown using a scanning $25 \mathrm{keV} \mathrm{He}^{+}$beam at normal incidence and for currents between 0.1 and $4.5 \mathrm{pA}$. The beam dwell time was varied between 5 and $1000 \mu$ s for a dose between 2.0 and $6.0 \mathrm{pC}$ per pillar. The current was regulated via the helium gas pressure in the source; thus ensuring 


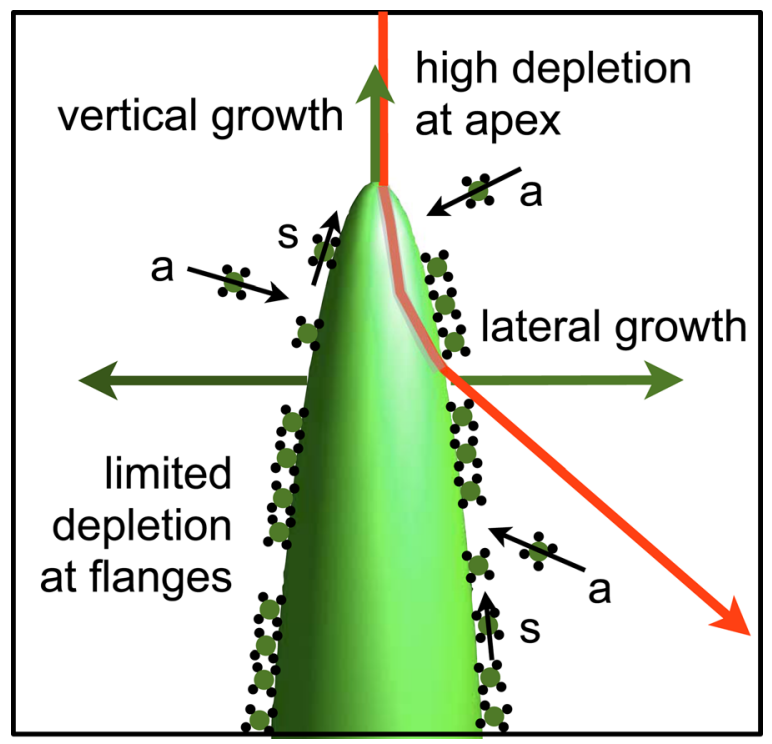

FIG. 1. (Color online) Growth of a pillar by beam induced deposition; vertical growth occurs mainly at the apex, where precursor depletion is high due to the high intensity of incoming ions and secondary electrons (SE1s). At the flanges, mainly lateral growth occurs, where depletion is limited due to the moderate intensity of forward scattered ions and secondary electrons (SE2s). Precursor refreshment occurs via adsorption ('a') or surface diffusion ('s').

a constant beam diameter. The number of pillars in an array was such that the beam return, or refresh, time was $1000 \mu \mathrm{s}$, unless stated otherwise. Thus, for the 5- $\mu$ s dwell time experiment, the number of pillars was 200 , whereas for the $1000-\mu \mathrm{s}$ one, it was 2. Additionally, nanopillars were grown with a continuous beam in which the dwell time was equal to the total deposition time. In one set of growth experiments, the voltage of the final lens was varied. The corresponding beam widths were derived from an independent measurement of the blur in the images of the edges in a grid. The ion beam was moved from one array site to another by electrostatic deflections with a bandwidth of $1 \mathrm{MHz}$. The deposits were imaged by subsequent HIM with the same beam current and at a $30^{\circ}$ sample tilt. We did not pursue any other analytical techniques.

\section{MODELING}

The pulsed He-IBID growth of the nanopillars was simulated using the "EnvisION" Monte Carlo ion beam induced deposition code $^{6}$ which is based on a previously developed electron beam induced deposition (EBID) simulation ${ }^{7,8}$ and a revision of the "IoniSE" ion-solid/secondary electron beam Monte Carlo routine. ${ }^{9}$ For a detailed description see Ref. 6. Briefly, the input parameters used are: beam energy $25 \mathrm{keV}$; ion currents of 0.7 and $4.5 \mathrm{pA}$; a Gaussian beam shape with a full-width-at-half-maximum of $1 \mathrm{~nm}$, unless stated otherwise; a composition of $20 \% \mathrm{Pt}$ and $80 \% \mathrm{C}$ with a density of $4.5 \mathrm{~g} / \mathrm{cm}^{3} ;^{3}$ a voxel size, $d$, of $0.48 \mathrm{~nm}$; and a total ion induced secondary electron yield of 2.9 electrons/ion for a flat $\mathrm{Pt}_{20} \mathrm{C}_{80}$ substrate, equal to the yields for both pure $\mathrm{C}$ and pure Pt. The localized $\left(\mathrm{CH}_{3}\right)_{3} \mathrm{Pt}\left(\mathrm{C}_{\mathrm{P}} \mathrm{CH}_{3}\right)$ pressure is $1.3 \times 10^{-2} \mathrm{mbar}^{3}{ }^{3}$ corresponding to a flux, $J$, of 11,000 ions $/ \mathrm{nm}^{2} / \mathrm{s}$. The sticking coefficient, $s$, of this molecule on the substrate and on the surface of the growing deposit is assumed to be $0.1 .^{10,11}$ The adsorption time, $\tau_{a}\left(=1 / s J d^{2}\right)$, is $4 \mathrm{~ms}$. We used an electron and ion beam induced dissociation cross-section as a function of energy, as presented in Ref. 3.

To simulate the pulsed beam conditions, each dwell time cycle was run for the number of ions $(=$ current $\times$ dwell time/ $1.6 \times 10^{-19} \mathrm{C}$ ) with the dynamic gas routine operating. ${ }^{7.8}$ After the last ion of each dwell cycle, the surface was re-set to the assigned equilibrium coverage (either $100 \%$ or $2 \%$, as will be described later) to cut short the long refresh time. The adsorption rate during the dwell cycle itself is described by a Langmuir isotherm and the kinetic theory of gases, where the flux on the surface is updated after each ion to mimic the continuous adsorption. Three situations were studied: 1) no spontaneous desorption, nor surface diffusion, which implied in a steady state coverage, $n_{e}$, of unity before the start of each dwell; 2) spontaneous desorption, with a desorption time, $\tau_{d}$, of $70 \mu \mathrm{s}$ resulting in a steady state coverage, $n_{e}$, of $2 \%$ (in equilibrium: $\left.s J=n_{e} d^{2} / \tau_{d} ; 3\right)$ spontaneous desorption and surface diffusion, with a desorption time, $\tau_{d}$, of $70 \mu \mathrm{s}$ and a diffusion constant, $D$, of $1 \times 10^{-9} \mathrm{~cm}^{2} / \mathrm{s}$.

\section{RESULTS AND DISCUSSION}

\section{A. Refresh-time and dwell-time dependences}

Figure 2 shows the height and width of pillars grown with different refresh times. The inserted HIM images show three example pillars at their corresponding refresh times. Although all other conditions were kept equal, the shapes of the pillars are different; specifically, pillars grown with refreshment are taller than those grown without. Moreover, if the refresh time is long $(5 \mathrm{~ms})$, the pillars are relatively broad. In general, a short refresh leads to a rapid increase in pillar height and a slight decrease in width (phase I), as compared to no refresh at all. At refresh times between 0.5 and $2 \mathrm{~ms}$ (phase II), the pillar height and width stabilize, whereas

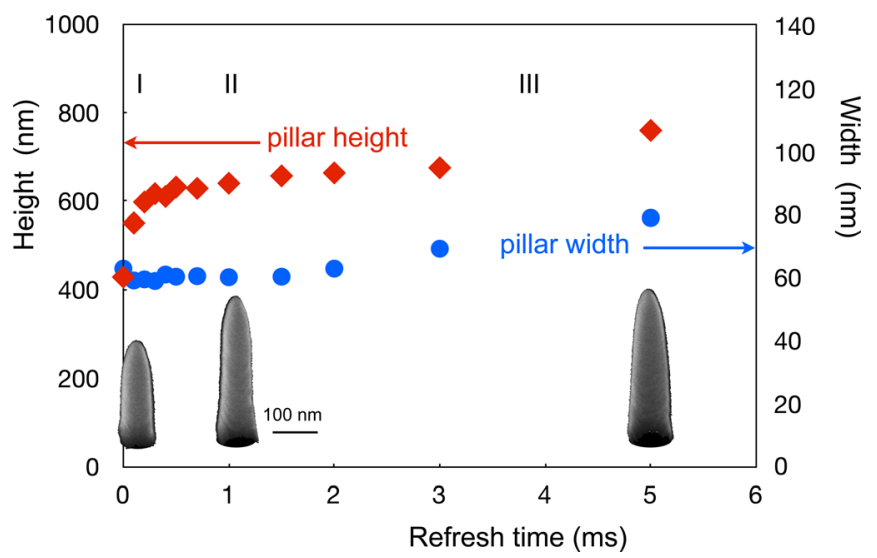

FIG. 2. (Color online) Pillar height and width as function of the refresh time (4.5 pA, $25 \mathrm{keV} \mathrm{He}{ }^{+}, 6 \mathrm{pC}, 100 \mu$ s dwell time.) Images of three typical pillars are shown at their respective refresh times. Three phases can be discerned: (I) a short refreshment enhances vertical growth and slightly reduces the pillar width as compared to no refreshment; (II) the height and width of the pillars are not affected by small changes in the refresh time; (III) both pillar height and width increase with refresh time. 


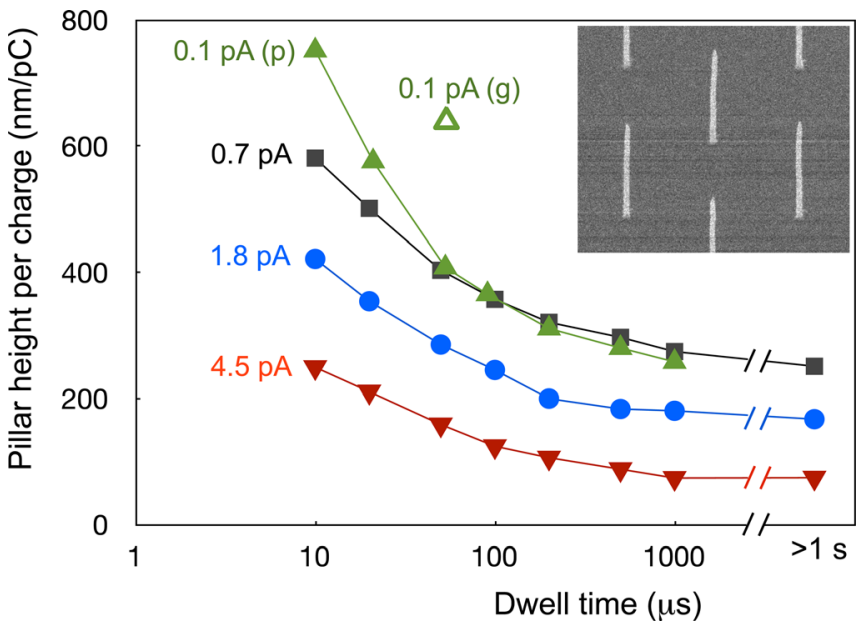

FIG. 3. (Color online) Vertical growth rate as function of beam dwell time and current $(25 \mathrm{keV}, 1 \mathrm{~ms}$ refresh time). The $0.1 \mathrm{pA}$ data point (open triangle) is discussed in Sec IV D. The inset is a HIM image of a few pillars of an array, taken at a $30^{\circ}$ sample tilt; the distance between the pillars is $1 \mu \mathrm{m}$.

at refresh times beyond $2 \mathrm{~ms}$ (phase III), both the height and the width continue to increase. The presence of two phases of increase suggests that there are two different mechanisms of precursor delivery or two relevant time- or distance-scales present. Because the pillar dimensions are insensitive to small variations in the refresh time during the 0.5 -to- $2 \mathrm{~ms}$ phase, all subsequent experiments have been performed with a fixed refresh time of $1 \mathrm{~ms}$.

The three subsequent figures show the vertical growth rate (pillar height per picoCoulomb; see Fig. 3), the pillar width (see Fig. 4), and the volumetric growth rate (volume per incident ion; see Fig. 5) for various dwell times and beam currents. Lower currents and shorter dwell times result in higher pillars (Fig. 3). With decreasing dwell time, the pillar width is at first constant, however, it increases below $100 \mu \mathrm{s}$. In general, the widths are larger at higher currents, except for the $0.1 \mathrm{pA}$ data. The volumetric growth rate (Fig. 5) shows a rapid increase with decreasing current and decreasing dwell time.

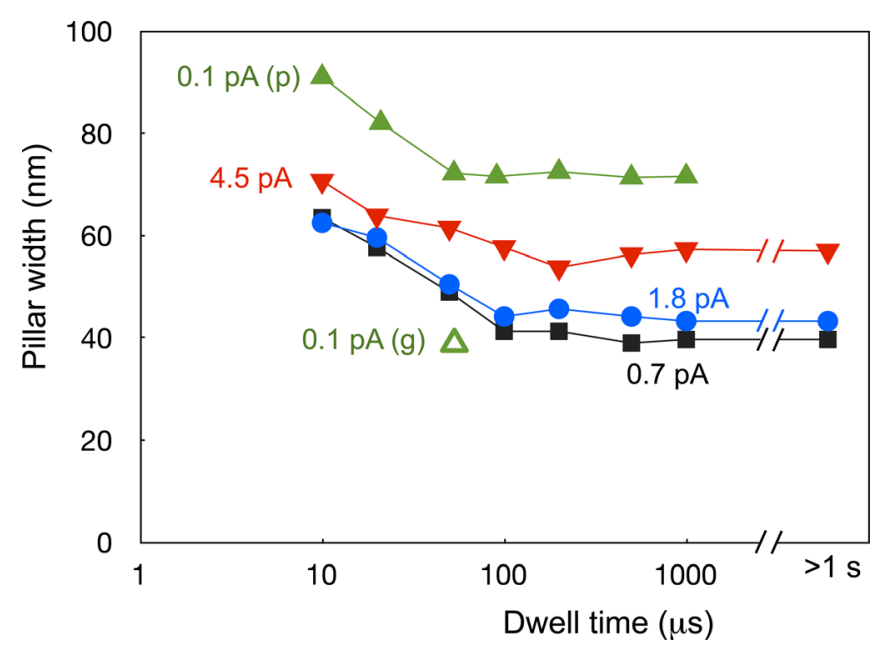

FIG. 4. (Color online) Pillar width as function of beam dwell time and current ( $25 \mathrm{keV}, 1 \mathrm{~ms}$ refresh time). The $0.1 \mathrm{pA}$ data point (open triangle) is discussed in Sec. IV D.

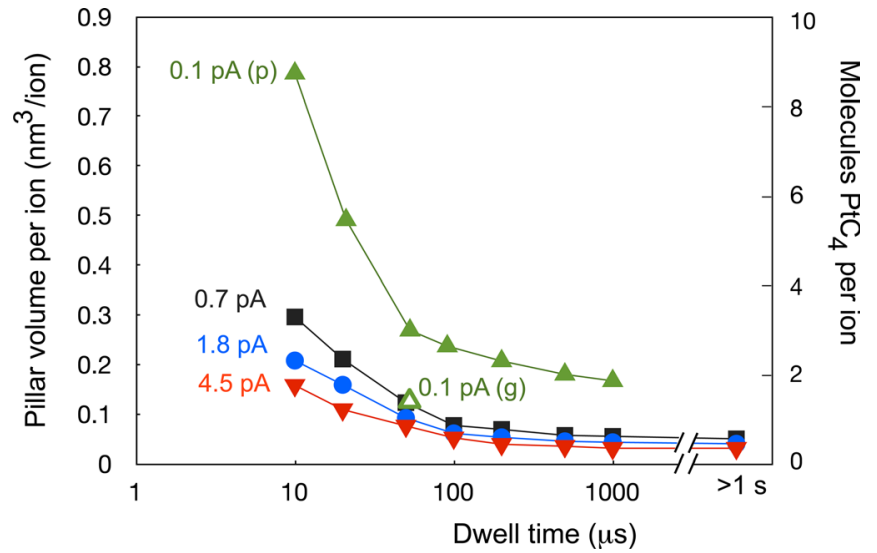

FIG. 5. (Color online) Volumetric growth rate as function of beam dwell time and current $(25 \mathrm{keV}, 1 \mathrm{~ms}$ refresh time). The $0.1 \mathrm{pA}$ data point (open triangle) is discussed in Sec. IV D. The vertical scale at right is derived from the volume, assuming a composition of $\mathrm{Pt}_{20} \mathrm{C}_{80}$ and a density of $11 \mathrm{PtC}_{4}$ molecules $/ \mathrm{nm}^{3}$.

Although previous work under similar conditions ${ }^{2,3,12}$ showed that the growth was entirely or predominantly reaction-rate limited, the increase shown in Fig. 5 is a strong indication of precursor-supply-rate-limited growth.

\section{B. Beam-size and -scanning dependences}

Ideally, the incident ion beam has zero-width, appears instantly at a site, and disappears instantly after a certain dwell time. In reality, the beam has a finite width and it requires a finite time to move the beam to and from the sites. As a consequence, the centers of growth are not limited to the points of the array and, hence, pillars might deviate in shape and size from the ideal ones. Figure 6 shows the measured dependence of pillar dimensions on the beam width. We see higher deposition rates for a defocused beam, similarly to the observation by Plank et al. in the EBID experiments. ${ }^{13}$ Remarkably, an increase of the full-width-at-halfmaximum from ' 0 ' (in reality $\sim 1 \mathrm{~nm}$ ) to, e.g., $10 \mathrm{~nm}$ results

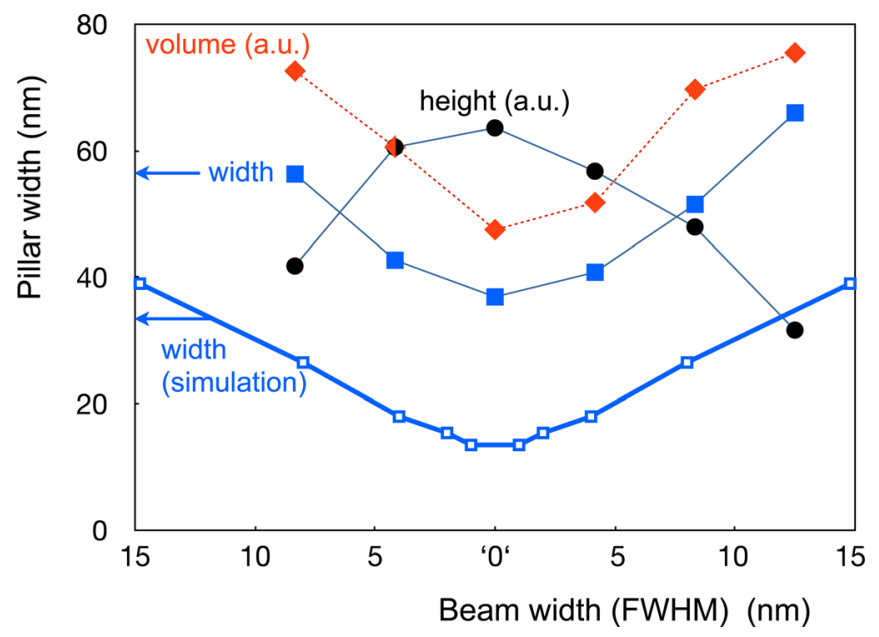

FIG. 6. (Color online) Volume, width, and height of pillars as a function of beam width. The beam width was varied by changing the focusing lens. The vertical scale refers to the width data only; ' 0 ' means as small as possible, in reality $\sim 1 \mathrm{~nm}$. The simulated width is discussed in Sec. IV C. 

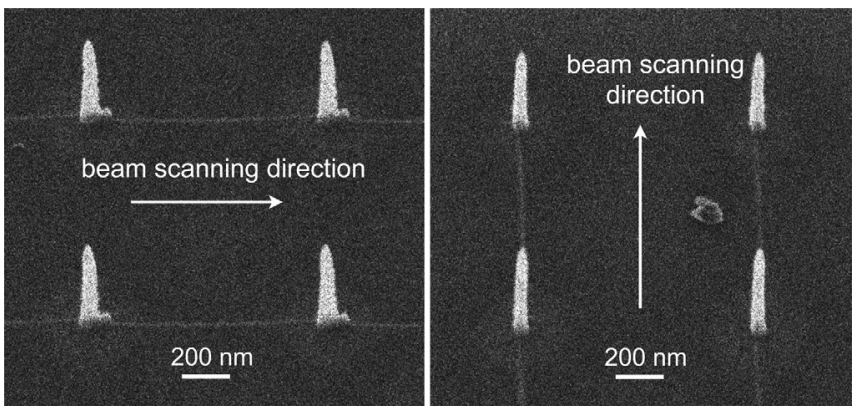

FIG. 7. Pillars from the same set, viewed from two perpendicular directions $\left(0.7 \mathrm{pA}, 20 \mu\right.$ s dwell time, $30^{\circ}$ sample tilt). The asymmetry in shape is thought to be caused by the finite $(\sim 1-10 \mu \mathrm{s})$ settling time of the ion beam.

in a pillar broadening from 40 to $60 \mathrm{~nm}$. Apparently, the intrinsic width of growth $(40 \mathrm{~nm})$ and the beam width do not add quadratically (this would be $42 \mathrm{~nm}$ ), and not even linearly (this would be $50 \mathrm{~nm}$ ). A similarly strong dependence on beam width has been observed by Ebm et al. in the IBID fabrication of more complex structures. ${ }^{14}$

Figure 7 shows pillars viewed from two different directions. Clearly, the pillars are not symmetric. In the beam movement direction they are wider than in the perpendicular direction. Moreover, there is a tiny hump at the foot of the pillar and between two successive pillars, there is a connecting line. Presumably, these irregularities are related to the relatively low speed and high inaccuracies of the beam deflection.

\section{Simulation results}

Figure 8 is an example of an intermediate simulation result for the case without spontaneous desorption. Depicted in Fig. 8 is the calculated coverage at the end of a dwell cycle for a high current with a long dwell time and a low current with a short dwell time, resulting in high and low depletion, respectively. For these simulations the equilibrium

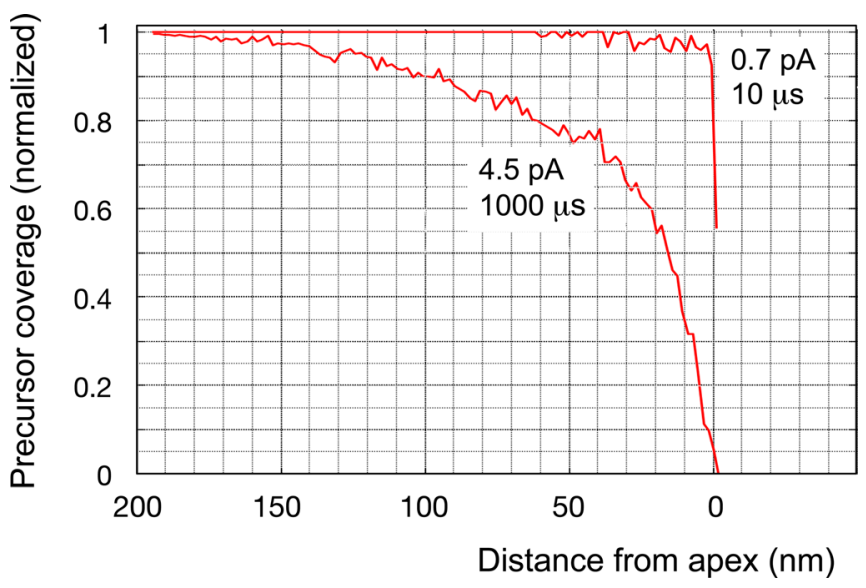

FIG. 8. (Color online) Calculated precursor coverage as function of the distance from the pillar apex for high ( $4.5 \mathrm{pA}$ current and $1000 \mu$ s dwell time) and low $(0.7 \mathrm{pA}$ and $10 \mu \mathrm{s}$ dwell time) depletion conditions. In the former case, depletion at the apex is high $(95 \%)$ and along the flange moderate $(<60 \%)$. In the latter case, depletion at the apex is moderate $(40 \%)$ and low at the flange $(<5 \%)$. Coverages are normalized to the value at long distance $(>200 \mathrm{~nm})$ from the apex.

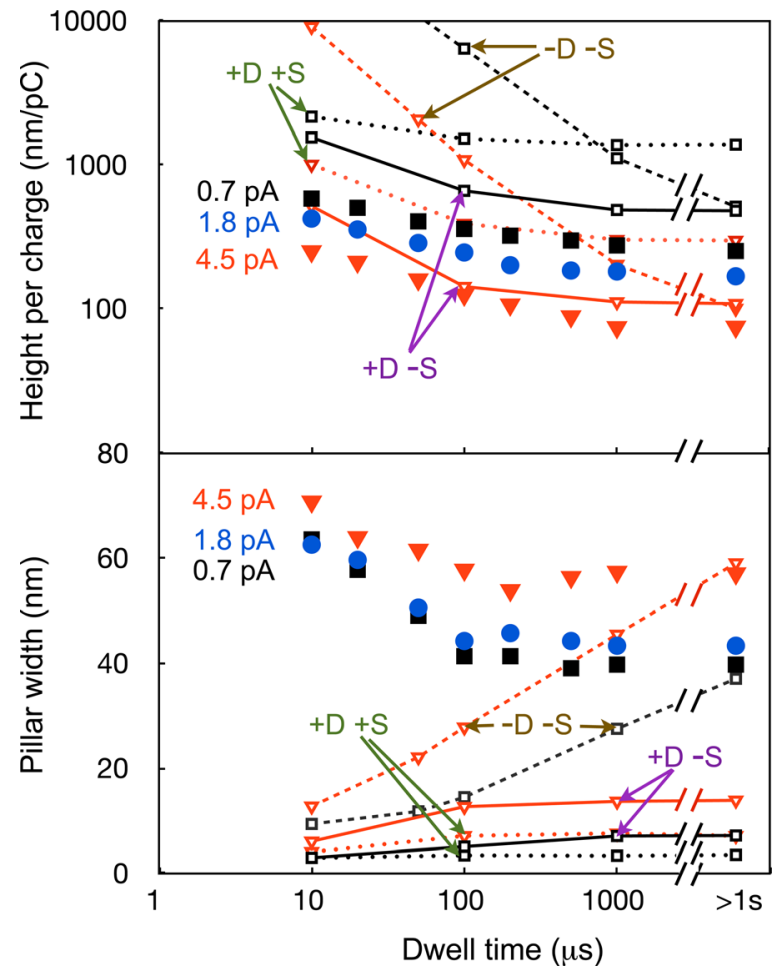

FIG. 9. (Color online) Experimental (filled data points) and calculated (open points with connecting lines) (a) vertical growth rates, and (b) pillar widths as function of dwell time and beam current. Simulations are for 0.7 and 4.5 pA without desorption and surface diffusion (dashed curves, ' $-\mathrm{D}-\mathrm{S}$ '); with a desorption time, $\tau_{d}$, of $70 \mu$ s but without surface diffusion (full curves, ' $+\mathrm{D}-\mathrm{S}$ '); and with the same desorption time and a diffusion constant $D$, of $10^{-9} \mathrm{~cm}^{2} / \mathrm{s}$ (dotted curves, ' $+\mathrm{D}+\mathrm{S}$ '). (Note that per condition, only 4 or 5 dwell times were simulated; the shown curves connect the data points.)

coverage at the beginning of each dwell cycle was unity. Each molecule present at the start of, or adsorbed during, a dwell cycle will remain unless it is decomposed by an ion or a secondary electron. As shown in Fig. 9(a) the 'no desorption no surface diffusion' $(-\mathrm{D}-\mathrm{S})$ simulations, viz., the dashed curves, predict the correct upward trend of increasing vertical growth rate with decreasing dwell time; however, it greatly exceeds the magnitude, particularly at short dwell times. The simulated pillar widths decrease with both decreasing current and decreasing dwell time, which is only partly consistent with the experimental results. As will be discussed later, we attribute the inconsistency for the dwelltime dependence to an artifact of the relatively slow beam steering. The decrease in the simulated pillar width in Fig. 9(b) with decreasing current and dwell time is due to the higher average precursor coverage at the pillar apex (see Figs. 1 and 8), which enhances the vertical growth rate and, hence, minimizes the lateral growth time. A range of coverage was explored and the simulations for the $70 \mu$ s desorption time resulted in a better quantitative agreement for the pillar height, see the full $(+D-S)$ curves in Fig. 9(a). This short desorption time is the same order of magnitude $(29 \mu \mathrm{s})$ as was estimated by Friedli et al. ${ }^{15}$ and the first-order desorption energy $(53 \mathrm{~kJ} / \mathrm{mol})$ estimated by Wnuk et al. ${ }^{16}$ The simulation data reproduce the trend of accelerated growth at short dwell times and saturated lower growth rates at longer 
dwell times. Interestingly, the pillars simulated with desorption are more narrow than without desorption which is, we think, due to the smaller difference in precursor density between the apex and the rest of the pillar surface when not only beam-induced, but also spontaneous, desorption is possible.

Figure 6 shows also the simulated pillar width as a function of beam size. Although all simulated widths are less than the experimental ones, there is the same strong dependence of the pillar width on beam size.

\section{Discussion}

Figure 1 shows a sketch of a growing IBID pillar. At the apex, a high-intensity ion beam enters the pillar. Interactions of the incident ions and their associated secondary electrons (SE1s) with adsorbed precursor molecules result in precursor decomposition and deposition of nonvolatile fragments; thus, growth of the pillar, mainly in the vertical direction. Most penetrating ions are deflected from their original trajectory and many of them exit from the pillar somewhere in the pillar beam interaction volume. Additionally, these forward scattered ions and their associated secondary electrons (SE2s) cause precursor decomposition and, hence, pillar growth, but now mainly in the lateral direction. Because the forward scattered ions are spread out over a large area, their density is relatively low. Obviously, no growth takes place beyond the maximal penetration depth of the ions, which is about $200 \mathrm{~nm}^{3,4}$

The high density of incident ions and their SE1s cause rapid precursor depletion at the pillar apex, whereas the density of forward scattered ions and SE2s at the flanges is much lower and, hence, depletion is much weaker there. The simulation at low current $(0.7 \mathrm{pA})$ and short dwell time $(10 \mu \mathrm{s})$ in Fig. 8 shows appreciable precursor depletion $(\sim 50 \%)$ at the apex but very little $(<2 \%)$ at the flanges. At a higher current $(4.5 \mathrm{pA})$ and a much longer dwell time $(1000 \mu \mathrm{s})$, depletion at the apex is almost complete $(>95 \%)$ and at the flanges it is substantial, ranging between $0 \%$ $150 \mathrm{~nm}$ below the apex to $\sim 70 \%$ close to the apex. The rapid increase in the vertical growth rate and the concurrent decrease in pillar width in Fig. 2 suggest that a refresh time of $\sim 100-200 \mu$ s is sufficient to counteract depletion at the apex. Precursor refreshment can occur both by surface diffusion and by adsorption. Because the area of the apex is small and because the gradient in coverage is large (see Fig. 8), diffusion is likely the dominant contributing mechanism here. Indeed, to cover a distance of the radius of the pillar apex $\left(r_{a}=9 \mathrm{~nm}^{3}\right)$ by diffusion in $100 \mu \mathrm{s}(=t)$, the diffusion constant, $D$, should be on the order of $10^{-9} \mathrm{~cm}^{2} / \mathrm{s}$ $\left(r_{a}=\sqrt{ } 4 D t\right)$. Although diffusion constants are not known for $\left(\mathrm{CH}_{3}\right)_{3} \mathrm{Pt}\left(\mathrm{C}_{\mathrm{P}} \mathrm{CH}_{3}\right)$, this value does not seem unrealistic. In phase III of Fig. 2, both the vertical growth rate and the pillar width increase with increasing dwell time. Although diffusion from the base of the pillar or from the substrate is possible, the involved time and length scales, respectively, several milliseconds and several hundreds of nanometers, are not compatible with a diffusion constant of $10^{-9} \mathrm{~cm}^{2} / \mathrm{s}$.
However, the slow rise in this phase III is consistent with the assumed adsorption time, $\tau_{a}$, of $4 \mathrm{~ms}$. The dotted $(+\mathrm{D}+\mathrm{S})$ curves in Fig. 9 show the pillar height and width simulated with a diffusion constant of $10^{-9} \mathrm{~cm}^{2} / \mathrm{s}$. Not surprisingly, diffusion dampens the drop in growth rate at the longer dwell times. Tentatively, we conclude that surface diffusion is the mechanism that replenishes the precursor molecules at the pillar apex and adsorption is the mechanism at the pillar flanges.

Shorter dwell times should result in less depletion; thus, faster vertical growth and narrower pillars. As mentioned in Sec. IV C, the experimental data show the opposite: the pillar width increases with decreasing dwell time. Figures 6 and 7 , which show a high sensitivity of the pillar shape on the beam conditions, give a clue to this finding. A slight defocusing or a relatively slow positioning of the beam results in pillar broadening. We conclude, therefore, that the observed increase in pillar width for decreasing dwell times below $100 \mu$ s is an experimental artifact, related to the slow and imprecise beam positioning. Unfortunately, this hypothesis can only be tested by replacing the used electronics by a faster version, which is an option that is not yet available on our instrument. Furthermore, all pillars produced with the $0.1 \mathrm{pA}$ current were too low and too broad. Focusing of a low-current beam is difficult due to the low signal-to-noise ratio and apparently not successfully conducted here. Indeed, an extra growth experiment at $0.1 \mathrm{pA}$ and $50-\mu$ s dwell time resulted in a tall and narrow pillar; see the open triangles in Figs. 3-5. This singular data point is consistent with the trend observed for the higher currents. Nevertheless, the illfocused beam at $0.1 \mathrm{pA}$ at short dwell times resulted in very high volumetric growth rates, up to $0.8 \mathrm{~nm}^{3} /$ ion, i.e., 20 times higher than achieved for a continuous and stationary beam (i.e., data points at dwell time of $>1 \mathrm{~s}$ ) and $\sim 15$ times higher than for a continuously scanning beam. ${ }^{2,12}$ Assuming a density of $11 \mathrm{PtC}_{4}$ molecules $/ \mathrm{nm}^{3}$ (Ref. 3) we conclude that per a single ion $\sim 9$ molecules can be decomposed, probably most of them by type-2 secondary electrons (SE2s). Note that, although we use the term $\mathrm{PtC}_{4}$ molecules, the deposited material is very likely not a $\mathrm{PtC}_{4}$ compound but an aggregate of small Pt-rich and C-rich particles. Furthermore, we did not measure the actual composition. Previous energy dispersive $\mathrm{x}$ ray work on a similar instrument with the same precursor yielded Pt contents between 9 and $20 \%,{ }^{2}$ which is comparable to EBID deposits with the same precursor. ${ }^{17}$

In Fig. 10, the same data are shown as in Fig. 3, however along the horizontal axis where the number of ions per dwell cycle is plotted $\left(=i \tau_{\mathrm{dwell}} / e\right)$. All data points lie roughly on a single curve. This observation implies that the vertical growth rate and, hence, depletion depend mainly on the number of ions per dwell cycle. The rapid initial decrease suggests that individual ions cause appreciable precursor depletion, although we do not know whether the adsorbed precursor molecules disappear due to decomposition only or to decomposition plus beam-induced desorption. There is a decrease in Fig. 10 by $15 \%$ between the first two 0.7 -pA data points, i.e., at 45 and 90 ions; thus a decrease of $0.33 \%$ per ion. Figure 5 shows that, typically, three $\mathrm{PtC}_{4}$ molecules are 


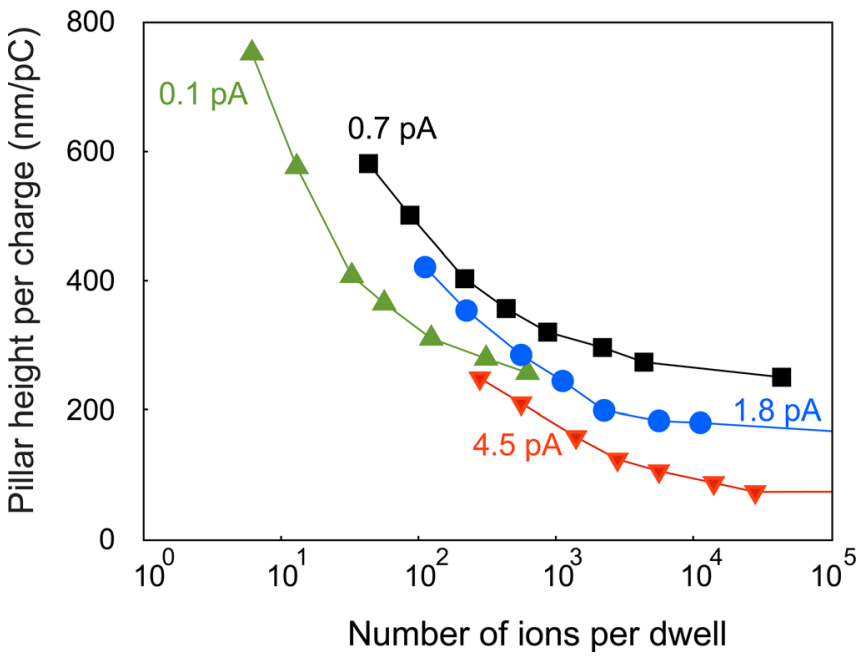

FIG. 10. (Color online) Vertical growth rate as a function of the number of ions per dwell. This figure suggests that depletion is mainly related to the number of incident ions per dwell cycle.

being deposited for this number of ions per cycle. Neglecting beam-induced desorption and assuming half of these three molecules decompose at the apex, either induced by primary ions or SE1s, we conclude that about 450 precursor molecules are available in the apex region at the start of each dwell cycle. If we take for the area of the spherical apex $2 \pi r_{a}^{2}$, where $r_{a}=9 \mathrm{~nm},{ }^{3}$ we arrive at an initial precursor density of $\sim 0.9 \quad \mathrm{PtC}_{4}$ molecules $/ \mathrm{nm}^{2}$. For a voxel size of $0.45 \mathrm{~nm}$, this is a surface coverage of $\sim 17 \%$. However, the actual $0.7-\mathrm{pA}$ pillar is more than $50 \%$ wider than the pillar mentioned in Ref. 3. Hence, the initial precursor density at the apex is probably at least twice as low. We conclude that the coverage deduced from the dwell-time dependence of the growth rate is higher, however, not really inconsistent with our simulation result of $2 \%$ in the short-desorption-time regime. However, the discrepancy between the measured and expected pillar widths at short dwell times introduces an extra uncertainty in our estimate.

\section{SUMMARY AND CONCLUSIONS}

This work shows that the growth of pillars by helium ion beam induced deposition is strongly dependent on the consumption of precursor molecules. If the beam is pulsed, refreshments are possible, leading to reduced depletion and thus, increased growth rates. Usually, faster vertical growth results in narrower pillars. ${ }^{8}$ However, if enhanced refreshments are achieved by dwell times shorter than the typical settling time of the beam deflection, inaccuracies in positioning arise, resulting in delocalized growth. Delocalized growth by inaccurate beam positioning or by beam defocusing results in a broadening of the pillars, which is much stronger than a simple convolution of the beam shape with the ideal pillar shape.

In general, low currents, short dwell times, and defocusing of the beam give the highest volumetric growth rates, expressed in volume grown per incident ion. Of course, high rates are not achieved by a generally enhanced supply of precursor molecules but instead by local reductions in depletion.
Especially when depletion in the beam impact region plus its surrounding SE1 area is avoided, growth rates can be high. We conlcude that there are, in fact, two different supplylimited regimes. One relates to depletion around the beam impact site and the other to the much larger area from which type- 2 secondary electrons are emitted. In this work, rates were measured up to 20 times the rate for nonpulsing conditions. However, the gain in volume comes at the cost of a loss in spatial precision. If one wants to grow structures at relatively high rates, a clever scheme of beam movements should be applied using fast and accurate beam deflections.

In general, pulsing experiments provide much detail in the basic physical and chemical processes of beam-induced deposition. We have applied Monte Carlo simulations, although not a complete fitting procedure, to uncover trends in the He-IBID growth. Despite some instrumental and procedural shortcomings, we were able to make estimates of the absolute precursor coverage, surface diffusion constant, and adsorption times.

\section{ACKNOWLEDGMENTS}

This research is part of NanoNed, a national research program on nanotechnology funded by the Ministry of Economic Affairs in The Netherlands. P.D.R. and D.A.S. acknowledge the support of the Semiconductor Research Corporation (Dan Herr, program manager). P.D.R. also acknowledges that a portion of this research was conducted at the Center for Nanophase Materials Sciences, which is sponsored by the Office of Basic Energy Sciences, U.S. Department of Energy, at the Oak Ridge National Laboratory.

${ }^{1}$ B. W. Ward, J. A. Notte, and N. P. Economou, J. Vac. Sci. Technol. B 24, 2871 (2006)

${ }^{2}$ C. A. Sanford, L. Stern, L. Barriss, L. Farkas, M. DiManna, R. Mello, D. J. Maas, and P. F. A. Alkemade, J. Vac. Sci. Technol. B 27, 2660 (2009).

${ }^{3}$ P. Chen, E. van Veldhoven, C. A. Sanford, H. W. M. Salemink, D. J. Maas, D. A. Smith, P. D. Rack, and P. F. A. Alkemade, Nanotechnology 21, 455302 (2010)

${ }^{4}$ P. F. A. Alkemade, P. Chen, E. van Veldhoven, and D. J. Maas, J. Vac. Sci. Technol. B 28, C6F22 (2010).

${ }^{5}$ J. D. Fowlkes and P. D. Rack, ACS Nano 4, 1619 (2010).

${ }^{6}$ D. A. Smith, J. D. Fowlkes, and P. D. Rack, Nanotechnology 19, 415704 (2008).

${ }^{7}$ D. A. Smith, D. Joy, and P. D. Rack, Nanotechnology 21, 175302 (2010).

${ }^{8}$ D. A. Smith, J. D. Fowlkes, and P. D. Rack, Nanotechnology 18, 265308 (2007).

${ }^{9}$ R. Ramachandra, B. Griffin, and D. Joy, Ultramicroscopy 109, 748 (2009).

${ }^{10}$ J. Puretz and L. W. Swanson, J. Vac. Sci. Technol. B 10, 2695 (1992).

${ }^{11} \mathrm{~S}$. T. Christensen, "Nanoscale investigation of platinum nanoparticles on $\mathrm{SrTiO}_{3}$ grown via physical vapor deposition and atomic layer deposition," Ph.D. thesis (Northwestern University, 2008).

${ }^{12} \mathrm{~S}$. Boden, Z. Moktadir, D. M. Bagnall, H. Mizuta, and H. N. Rutt, Microelectron. Eng. 88, 2452 (2011).

${ }^{13}$ H. Plank, C. Gspan, M. Dienstleder, G. Kothleitner, and F. Hofer, Nanotechnology 19, 485302 (2008).

${ }^{14}$ C. Ebm, G. Hobler, S. Waid, and H. D. Wanzenboeck, J. Vac. Sci. Technol. B 29, 11031 (2011).

${ }^{15}$ V. Friedli, C. Santschi, J. Michler, P. Hoffmann, and I. Utke, Appl. Phys. Lett. 90, 053106 (2007).

${ }^{16}$ J. D. Wnuk, J. M. Gorham, S. Rosenberg, W. F. van Dorp, T. E. Madey, C. W. Hagen, and D. H. Fairbrother, J. Phys. Chem. C 113, 2487 (2009).

${ }^{17}$ I. Utke, P. Hoffmann, and J. Melngailis, J. Vac. Sci. Technol. B 26, 1197 (2008). 